\title{
Indeterminacy, Underground Activities and Tax Evasion*
}

\author{
Francesco Busato $^{\dagger} \quad$ Bruno Chiarini ${ }^{\ddagger} \quad$ Enrico Marchetti ${ }^{\S}$
}

This Draft: January 27, 2010

\begin{abstract}
This paper introduces underground activities and tax evasion into a one sector dynamic general equilibrium model with aggregate external effects. The model presents a novel mechanism driving the self-fulfilling prophecies, which is characterized by well behaved (downward sloping) labor demand schedules. This mechanism differs from the customary one, and it is complementary to it. Compared to traditional labor market income, the income derived from underground labor activity is subject to a lower expected tax rate when considering both the probability of detection and the evasion penalty. During a belief-driven expansion, the household allocates more time to both traditional and underground labor supply. In equilibrium, this action serves to lower the effective labor tax rate faced by the household, thus providing stimulus to aggregate labor supply so as to make the initial expansion self-fulfilling. The mechanism here is akin to a "regressive tax"; the household's effective tax rate depends negatively on the level of total labor income. We argue that an underground sector, and the associated tax evasion, offer a good economic rationale for a regressive tax rate.
\end{abstract}

Journal of Economic Literature Classification Numbers: O40, E260

Keywords: Indeterminacy and Sunspots, Tax Evasion and Underground Activities.

\footnotetext{
${ }^{*}$ We are grateful to Torben M. Andersen, John B. Donaldson, Marco Maffezzoli, Luca Stanca, Claus Vastrup for many conversations and to the participants at Seminars at University of Aarhus, University of Rome "La Sapienza", at the III Workshop on Macroeconomic Dynamics at Bocconi University, at XIII Annual Symposium of the Society of Non Linear Dynamics and Econometrics at City University of London, and at the XLV Conference of the Italian Economic Association for comments on earlier drafts of the paper.

${ }^{\dagger}$ Corresponding Author. University of Naples "Parthenope", Department of Economic Studies, Via Medina 40, 80133 Naples, Italy. Tel. +39 081 5474734; Fax: +39 081 5474750. E-mail address: busato@uniparthenope.it.

${ }^{\ddagger}$ Department of Economic Studies, University of Naples Parthenope, Naples, Italy.

${ }^{\S}$ Department of Economic Studies, University of Naples Parthenope, Naples, Italy.
} 


\section{Introduction}

This paper introduces underground activities and tax evasion into a one sector dynamic general equilibrium model with external effects, and shows that these phenomena are a possible source of local indeterminacy of the equilibrium path. We present a one-sector dynamic general equilibrium model in which there are three agents: firms, households and a government; furthermore, there is one homogeneous consumption good and three production factors: regular labor, underground labor, and a capital stock. Government levies income taxes on regularly produced income flows, and labor taxes on regular labor services, and balances its budget (in expected terms) for each period. Firms and households, being subject to distortionary taxation, use the underground labor input to evade taxes. Government faces tax evasion originating from the underground sector, and coordinates strategy to address abusive tax evasion schemes.

The introduction of underground labor services eases the necessity of having an upward sloping labor demand schedules. ${ }^{1}$ In addition, a plausibly-calibrated underground sector can reduce the required degree on increasing returns for indeterminacy to around 1.4, as compared to a value of about 1.6 in a model without underground labor (recent estimates suggest that the United States economy returns to scale are no larger than 1.2 (see, among the others, Basu and Fernald [2], Sbordone [17], Jimenez and Marchetti [13]). Notice that the reduction of the aggregate degree of returns to scale is not the main goal of the paper, especially because the literature proposes several mechanisms capable of reducing the required degree of returns to scale for indeterminacy to figures even smaller than the actual one (cfr. Wen [20]; Benhabib and Farmer [4]; Guo and Lansing [11]; Perli [16]); the general idea behind these contributions is to add at least one additional dimension to the baseline model. Our main interest is in the theoretical mechanism operating under tax evasion.

The economic mechanism is triggered by the reallocation of resources to the underground sector to avoid the excess tax burden. This mechanism differs from the customary one, and it is complementary to it. It turns out that prophecies of a higher expected income, triggered by a sunspot shock, are self-fulfilled through a resource reallocation toward the underground labor services; this allows to evade taxes, and therefore to have additional resources (the tax wedge) for satisfying the higher desired consumption profile.

In other words, compared to traditional labor market income, the income derived from underground labor activity is subject to a lower expected tax rate when considering both the probability of detection and the evasion penalty. During a belief-driven expansion, the household allocates more time to both traditional and underground labor supply. In equilibrium, this action serves to lower the effective labor tax rate faced by the household, thus providing stimulus to aggregate labor supply so as to make the initial expansion self-fulfilling.

\footnotetext{
${ }^{1}$ There is no universal agreement on what defines the underground economy. Most recent studies use one of more of the following definitions: (a) unrecorded economy (failing to fully or properly record economic activity, such as hiring workers off-the-book); (b) unreported economy (legal activity meant to evade the tax code); (c) illegal economy (trading in illegal goods and services). Obviously, the difficulty in defining the sector extends to the estimation of its size. We are concerned with the size of the underground economy as encompassing activities which are otherwise legal but go unreported or unrecorded.
} 
The mechanism here is akin to a "regressive tax"; the household's effective tax rate depends negatively on the level of total labor income. We argue that an underground sector, and the associated tax evasion, offer a good economic rationale for a regressive tax rate. $^{2}$

The model is calibrated for the United States economy, where the size of underground economy ranges between 5 percent of GNP (Tanzi, [19]) and 9 percent of GDP (Schenider and Enste, [18]; Paglin [15]). Even though these figures are below the OECD countries average (17 percent, according to Schneider and Enste [18]), they still represent a significant amount of resources absconded from tax collection. Notice, more importantly, that even a tiny underground sector matters for inducing local indeterminacy of the equilibrium path; and the United States underground sector's size is above this threshold.

The paper is organized as follows. Section 2 details the model; Section 3 presents the topological properties of stationary state, discusses conditions for indeterminacy and describes the theoretical mechanism. Next Section 4, presents the model's implication for the overall level of returns to scale, and Section 5 checks the results' robustness through a sensitivity analysis. Finally Section 6 concludes, while proofs and derivations are included in the Appendix.

\section{The model}

\subsection{Firms}

Assume that there exists a continuum of firms, uniformly distributed over the unit interval. Production technology for the homogenous good uses three inputs: physical capital, regular labor services, and underground labor services. The production function of firm $j \in[0,1]$ reads:

$$
y_{t}^{j}=A_{t}\left(k_{t}^{j}\right)^{\alpha}\left(n_{M, t}^{j}\right)^{1-\alpha-\rho}\left(n_{U, t}^{j}\right)^{\rho}, \quad \alpha, \rho \in(0,1),
$$

where $k_{t}^{j}$ denotes capital stock, $n_{M, t}^{j}$ is regular labor, $n_{U, t}^{j}$ represents underground labor, and the quantity $A_{t}$ is an aggregate production externality (defined below). A different modelling option is that of Busato and Chiarini [5], who set up a two sector model with underground economy; as they show that the model has an interior solution for a standard parametrization, we think that the use of a one sector formulation as in (1) is not a strong assumption. The production function follows a "moonlighting production scheme", where underground labor services use the same capital stock that is used by regular labor. ${ }^{3}$ We could imagine, for example, that the same firm produces in the regular economy by day, and in the underground economy by night. The model implicitly assumes that firms always use some underground labor services. In this regard this model applies to

\footnotetext{
${ }^{2}$ As expected, the explicit introduction of distortionary taxation combined with these phenomena into a one-sector general equilibrium model allows to reduce the aggregate degree of increasing returns required for indeterminacy, and for having well behaved demand schedules for production inputs (in the sense that they slope down). It is well known, indeed, that regressive tax structures can ease the conditions needed for equilibrium indeterminacy in this class of models (see Guo and Lansing, [10]).

${ }^{3}$ see Bajada [1] and Cowell [6] for a detailed definition of moonlighting production scheme.
} 
an economy where there exists at least one firm hiring at least one worker in the underground labor market. We think, however, that this is still a general formulation, because it would be difficult to find economies without any form of tax evasion. In addition, official GDP estimates incorporate an estimate of the contribution produced by the underground sector.

The aggregate production externality $A_{t}$ is defined below:

$$
A_{t}=\underbrace{\left\{\left(K_{t}^{\alpha} N_{M, t}^{1-\alpha-\rho}\right\}^{\eta}\right.}_{\text {Marshallian Ext. }} \underbrace{\left\{N_{U, t}^{\rho}\right\}^{\zeta}}_{\text {Underground Labor Ext. }} \quad \zeta, \eta \geq 0, \zeta \gtrless \eta,
$$

where capital letters denote aggregate quantities (in a perfect foresight symmetric equilibrium; details below $).{ }^{4}$ We distinguish between the "regular" externality $\left\{\left(K_{t}^{\alpha} N_{M, t}^{1-\alpha-\rho}\right\}^{\eta}\right.$ that is related to the well known Marshallian effect, and the underground labor external effect $\left\{N_{U, t}^{\rho}\right\}^{\zeta}$; as the model has just one homogenous good, $\zeta$ represents an "input specific" externality, rather than a sector specific one. Once we allow for labor heterogeneity at the firm and individuals' level, it is natural to do the same at the aggregate one. ${ }^{5}$ The underground externality can be tied, for example, to the fact that there are young pensioners entering the underground labor market. These workers have a high productivity, but choose to work in the underground sector for an additional income, while keeping their pensions at the same time. Needless to say, this would not be possible if they were hired under a regular labor contract. There is more. A significant part of the underground labor force is made of immigrants, who try to be as productive as they can, in order to be hired as (transformed into) regular workers. Immigrants usually enter relatively more developed countries with temporary visas, which are converted into permanent ones only when they prove to be regularly employed. Section 4.2 discusses in more details how to pin down precise values for $\eta$ and $\zeta$.

It is finally interesting to anticipate that the economic mechanism of this model and the intuition would be preserved even if the underground specific external effect $(\zeta)$ were set to zero. What matters here is the very existence of a underground sector $(\rho>0)$ that allows, intuitively speaking, to better use the regular-sector external effect.

As firms are homogeneous, the overall level of output for a given (and equal for all firms) level of input utilization is given by:

$$
Y_{t}=A_{t} \int_{j}\left\{\left(k_{t}^{j}\right)^{\alpha}\left(n_{M, t}^{j}\right)^{1-\alpha-\rho}\left(n_{U, t}^{j}\right)^{\rho}\right\} d j=K_{t}^{\alpha(1+\eta)} N_{M, t}^{(1-\alpha-\rho)(1+\eta)} N_{U, t}^{\rho(1+\zeta)} .
$$

Increasing returns to scale are a pure aggregate phenomenon (as Eq. (1) suggests), and returns to scale are constant at the firms' level, as each firm takes $K_{t}, N_{M, t}$ and $N_{U, t}$ as given for all $t=1,2, \ldots, T, \ldots$

Firms try to evade taxes on labor services by allocating labor demand to underground labor

\footnotetext{
${ }^{4}$ The aggregate value of a variable $z_{j}$ is defined as: $Z=\int_{0}^{1}\left(z_{j}\right) d j$.

${ }^{5}$ This formulation also adds generality to the analysis: when $\eta=\zeta$ and there are neither tax evasion nor distortionary taxation, the model reduces to Farmer and Guo's one.
} 
market. Firms, however, may be detected evading, with exogenous probability $p \in(0,1)$, and forced to pay the statutory tax rates on labor $\left(\tau_{N}\right)$, increased by a surcharge factor, $s>1$, applied to the standard tax rate. We implicitly assumes that firms always try to evade taxes by reallocating some labor demand to the underground labor. It is a consequence of the production technology: in order to have nonzero production, $N_{U, t}^{j}$ must be positive in equilibrium. For studying an economy without tax evasion, we should impose $\rho=0$; but in this case, the model would reduce to a standard one sector economy without labor market segmentation.

When a firm is not detected evading (with probability $1-p$ ), its profits are denoted with $\pi_{N D, t}^{j}$. If detected evading (with probability $p$ ), we denote firm's profits as $\pi_{D, t}^{j}$; both are defined below, after normalizing the output price to unity:

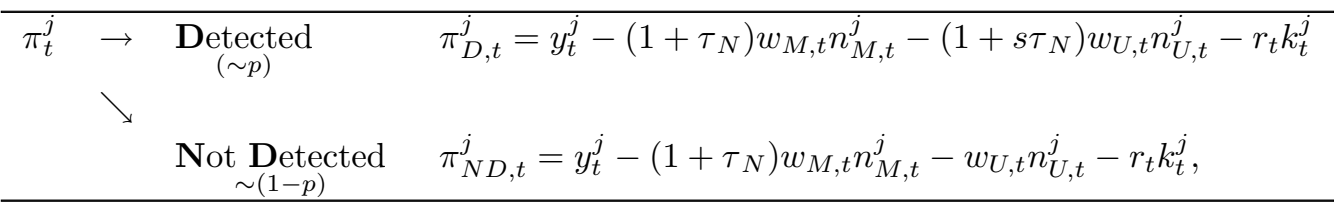

where $w_{M, t}$ and $w_{U, t}$ denote the regular and the underground sector wages, $r_{t}$ is capital remuneration rate. Finally, expected profits are $E \pi_{t}^{j}=(1-p) \pi_{N D, t}^{j}+p \pi_{D, t}^{j}$ :

$$
E \pi_{t}^{j}=y_{t}^{j}-\left(1+\tau_{N}\right) w_{M, t} n_{M, t}^{j}-\left(1+p s \tau_{N}\right) w_{U, t} n_{U, t}^{j}-r_{t} k_{t}^{j}
$$

where $E$ denotes an expectation operator. Underground activities lead to fines if caught; the parameter $s>1$ represents the surcharge on the statutory tax rate that a firm, detected employing workers in the underground labor market, must pay. The model also incorporates an extra disutility on the consumers' side of the economy, when supplying labor into the underground economy (more details to come).

As markets are competitive, the representative firm's behavior is described by the first order conditions for the (expected) profit maximization, with respect to $k_{t}^{j}, n_{M, t}^{j}$ and $n_{U, t}^{j}$ :

$$
\left\{\begin{array}{l}
\frac{\partial y_{t}^{j}\left(A_{t}\right)}{\partial k_{t}^{j}}=r_{t} \\
\frac{\partial y_{t}^{j}\left(A_{t}\right)}{\partial n_{M, t}^{j}}=\left(1+\tau_{N}\right) w_{M, t} \\
\frac{\partial y_{t}^{j}\left(A_{t}\right)}{\partial n_{U, t}^{j}}=\left(1+s p \tau_{N}\right) w_{U, t}
\end{array}\right\}
$$

Concavity of the production function (recall that firms take $A_{t}$ as a constant) ensures the existence of a unique solution. Before proceeding, notice that in this economy the labor heterogeneity mainly comes from the differential taxation scheme for regular and underground income flows, and from the consumers' side (below), the production technology being Cobb-Douglas. An alternative interesting formulation would specify the production technology as a nested Constant Elasticity of Substitution production function to study the substitutability between the regular inputs (regular labor and capital) and underground labor services. In this case, however, it would not be possible to analytically 
derive the conditions for indeterminacy. We think that one of the welcomed features of this scheme is indeed to be have conditions for indeterminacy that can be nicely interpreted from an economic perspective.

\subsection{Households}

Suppose that there exists a continuum of households, uniformly distributed over the unit interval. The $h$-th household's preferences are represented by the following momentary utility function:

$$
\mathcal{V}_{t}^{h}=\log \left(c_{t}^{h}\right)-B_{0}\left(n_{M, t}^{h}+n_{U, t}^{h}\right)-B_{1}\left(n_{U, t}^{h}\right), \quad B_{0}, B_{1} \geq 0
$$

where $c_{t}^{h}$ denotes household's consumption flow, $n_{M, t}^{h}$ and $n_{U, t}^{h}$ denote regular and underground labor supplies; the quantity $B_{0}\left(n_{M, t}^{h}+n_{U, t}^{h}\right)$ represents the overall disutility of working, while the last term, $B_{1}\left(n_{U, t}^{h}\right)$, reflects the idiosyncratic cost of working in the underground labor market. Specifically, this cost may be associated with the lack of any social and health insurance in the underground sector; although there exist perfect substitutability across labor markets (in the sense that there are no adjustment costs while transferring labor supply (demand) from a market to an other) each labor market has its own peculiar characteristics that instantaneous utility function tries to capture with regard to consumer's behavior. The paper assumes perfectly elastic labor supply schedules. This is a customary assumption, commonly accepted in this literature (see among the many Farmer and Guo [7]); it also allows to find a closed form for the stationary state.

The representative household evades income taxes by reallocating labor services from regular to underground labor markets. Underground-produced income flows $w_{U, t} n_{U, t}^{h}$ are, therefore, not subject to the distortionary income tax rate $\tau_{Y}$, as the budget constraint below suggests: ${ }^{6}$

$$
c_{t}^{h}+k_{t+1}^{h}=\left(1-\tau_{Y}\right)\left(w_{M, t} n_{M, t}^{h}+r_{t} k_{t}^{h}\right)+w_{U} n_{U, t}^{h}+(1-\delta) k_{t}^{h},
$$

where $k_{t+1}^{h}$ is next period capital stock, and $\delta$ denotes a quarterly capital stock depreciation rate.

Imposing a constant subjective discount rate $0<\beta<1$, and defining $\mu_{t}^{h}$ as the costate variable, the representative household maximizes the Lagrangian $\mathcal{L}_{0}^{h}$ :

$$
\begin{aligned}
\max _{\left\{c_{t}^{h}, n_{M, t}^{h}, n_{U, t}^{h}, k_{t+1}^{h}\right\}_{t=0}^{\infty}} \mathcal{L}_{0}^{h}= & E_{0} \sum_{t=0}^{\infty} \beta^{t} \mathcal{V}_{t}^{h}+ \\
& +E_{0} \sum_{t=0}^{\infty} \mu_{t}^{h}\left\{\left(1-\tau_{Y}\right)\left(w_{M, t} n_{M, t}^{h}+r_{t} k_{t}^{h}\right)+w_{U} n_{U, t}^{h}-k_{t+1}^{h}+(1-\delta) k_{t}^{h}-c_{t}^{h}\right\} .
\end{aligned}
$$

Optimal household's choice is characterized by the following first order conditions:

\footnotetext{
${ }^{6}$ The tax rate $\tau_{Y}$ is not a legitimate income tax, because part of produced income is not taxed. Specifically, it is an income tax that applies to declared income flows. In addition, a value added tax would be part of an optimal tax policy in the presence of underground activities (although this tax also can be evaded). We abstract, however, from this tax and we leave it for further investigations.
} 


$$
\left\{\begin{array}{c}
\beta^{t}\left(c_{t}^{h}\right)^{-1}=\mu_{t}^{h} \\
\beta^{t} B=\mu_{t}^{h}\left(1-\tau_{Y}\right) w_{M, t} \\
\beta^{t} B_{0}+\beta^{t} B_{1}=\mu_{t}^{h} w_{U, t} \\
E_{t}\left\{\mu_{t+1}^{h}\left[(1-\delta)+\left(1-\tau_{Y}\right) r_{t+1}\right]\right\}=\mu_{t}^{h} \\
\lim _{T \rightarrow \infty} E_{0} \mu_{T}^{h} k_{T}^{h}=0 .
\end{array}\right\}
$$

\subsection{Government}

The government budget reads

$$
\tau_{Y}\left(w_{M, t} N_{M, t}+r_{t} K_{t}\right)+s p \tau_{N} w_{U, t} N_{U, t}+\tau_{N} w_{M, t} N_{M, t}=G_{t}
$$

where the left-hand side denotes expected government revenues that are allocated to aggregate government expenditure $G_{t}$, which is is assumed to be wasteful. Capital letters denote aggregate equilibrium quantities (defined below). Government balances its budget in expected terms since tax revenues collected from the underground side corporate sector also depend on the probability of being detected $p$.

\subsection{Perfect foresight equilibrium}

We focus on a perfect foresight equilibrium in which households maximize utility and firms make zero profits. In equilibrium the aggregate consistency requires that $y_{t}=Y_{t}, k_{t}=K_{t}, n_{M, t}=N_{M, t}$, $n_{U, t}=N_{U, t}, c_{t}=C_{t}$, where lower capital letters denote individual equilibrium quantities, and capital letters denote aggregate equilibrium quantities. As a result, the first order conditions characterizing the equilibrium are given by:

$$
\begin{gathered}
B_{0}=\left(C_{t}\right)^{-1}\left(1-\tau_{Y}\right) \frac{(1-\alpha-\rho)}{\left(1+\tau_{N}\right)} \frac{Y_{t}}{N_{M, t}} \\
B_{0}+B_{1}=\left(C_{t}\right)^{-1} \frac{\rho}{1+s p \tau_{N}} \frac{Y_{t}}{N_{U, t}} \\
\left(C_{t+1}\right)^{-1}\left((1-\delta)+\left(1-\tau_{Y}\right) \alpha \frac{Y_{t+1}}{K_{t+1}}\right) \beta=\left(C_{t}\right)^{-1} \\
K_{t+1}=\left(\left(1-\tau_{Y}\right)\left(\frac{(1-\alpha-\rho)}{\left(1+\tau_{N}\right)}+\alpha\right)+\frac{\rho}{1+s \rho \tau^{N}}\right) K_{t}^{\phi_{1}} N_{M, t}^{\phi_{2}} N_{U, t}^{\phi_{3}}+(1-\delta) K_{t}-C_{t} \\
\lim _{t \rightarrow \infty}\left(C_{t}\right)^{-1} K_{t}=0
\end{gathered}
$$

where $Y_{t}=K_{t}^{\phi_{1}} N_{M, t}^{\phi_{2}} N_{U, t}^{\phi_{3}}, \phi_{1}=\alpha(1+\eta), \phi_{2}=(1-\alpha-\rho)(1+\eta)$ and $\phi_{3}=(1+\zeta) \rho$. 


\subsection{Stationary state}

Proposition 1 shows that the model has a unique stationary state for capital stock, and unique values for equilibrium regular and underground labor services.

Proposition 1 There exists a unique stationary capital stock $K^{\star}>0$, and a unique stationary equilibrium for regular labor supply $N_{M}^{\star}>0$, and a unique stationary ratio $\left(\frac{N_{U}}{N_{M}}\right)^{\star}$ such that:

$$
\begin{aligned}
K^{\star} & \simeq\left(\frac{\left(1-\tau_{Y}\right) \alpha}{\beta^{-1}-1+\delta}\right)^{\frac{1}{1-\alpha(1+\eta)}}\left(\frac{1+\tau_{N}}{1+s p \tau_{N}} \frac{B_{0} \rho(1-\alpha-\rho)^{-1}}{\left(B_{0}+B_{1}\right)\left(1-\tau_{Y}\right)}\right)^{\frac{\rho(1+\zeta)}{1-\alpha(1+\eta)}}\left(N_{M}^{\star}\right)^{\frac{(1-\alpha)(1+\eta)}{1-\alpha(1+\eta)}} \\
N_{M}^{\star} & \simeq \frac{(1-\alpha-\rho)}{B_{0}\left(\left(1-\tau_{Y}\right)\left(\bar{\Psi}_{M}+\frac{\beta^{-1}-1+\delta}{1-\tau_{Y}}\right)+\bar{\Psi}_{U}-\delta\right)}\left(\frac{\beta^{-1}-1+\delta}{\alpha\left(1+\tau_{N}\right)}\right) ; \\
\left(\frac{N_{U}}{N_{M}}\right)^{\star} & =\frac{B_{0} \rho(1-\alpha-\rho)^{-1}}{\left(B_{0}+B_{1}\right)\left(1-\tau_{Y}\right)} \frac{1+\tau_{N}}{1+p s \tau_{N}},
\end{aligned}
$$

where $\frac{(1-\alpha-\rho)}{\left(1+\tau_{N}\right)} \frac{\beta^{-1}-1+\delta}{\left(1-\tau_{Y}\right) \alpha}=\bar{\Psi}_{M}$ and $\frac{\rho}{\left(1+s p \tau_{N}\right)} \frac{\beta^{-1}-1+\delta}{\left(1-\tau_{Y}\right) \alpha}=\bar{\Psi}_{U}$.

Proof. See Appendix.

\section{Topological properties and an original theoretical mechanism}

\subsection{Topological properties}

To study topological properties of the stationary state derived in Proposition 1, we arrange the system of linearized equations so to obtain a planar dynamical system in the state vector $\widehat{S}_{t}=\left(\widehat{K}_{t} ; \widehat{C}_{t}\right)$ (hat-variables denote percentage deviations from their steady state values):

$$
E_{t} \widehat{S}_{t+1}=\mathcal{W} \widehat{S}_{t}+\Omega \mathcal{E}_{t+1}
$$

where $\mathcal{E}_{t+1}$ is a $2 \times 1$ vector of one step ahead forecasting errors satisfying $E_{t} \mathcal{E}_{t+1}=0$, and $\Omega$ is a coefficient matrix. Its first element $\widehat{K}_{t+1}-E_{t} \widehat{K}_{t+1}$ equals zero, since $K_{t+1}$ is known at period $t$; denote the second element with $\tilde{\varepsilon}_{c}=\widehat{C}_{t+1}-E_{t} \widehat{C}_{t+1}$.

We now introduce the following standard definition.

Definition 1 The stationary state $K^{\star}$ is called locally indeterminate if there exists $\epsilon>0$ such that from any $K_{0}$ belonging to $\left(K^{\star}+\epsilon, K^{\star}-\epsilon\right)$ there are infinitely many equilibrium paths converging to the steady state.

Now, when the model has a unique equilibrium (i.e., one of the eigenvalues of $\mathcal{W}$ lies outside the unit circle), the optimal decision rule for consumption does not depend on the forecasting error, $\tilde{\varepsilon}_{c}$. If both eigenvalues of $\mathcal{W}$ lie inside the unit circle, however, the model is indeterminate in the sense that any value of $\widehat{C}_{t}$ is consistent with equilibrium given $\widehat{K}_{t}$. Hence, the forecasting error $\tilde{\varepsilon}_{c}$ can play 
a role in determining the equilibrium level of consumption. Under indeterminacy the decision rule for consumption at time $t$ takes the special form

$$
\widehat{C}_{t}=w_{21} \widehat{K}_{t-1}+w_{22} \widehat{C}_{t-1}+\omega_{2} \tilde{\varepsilon}_{c, t},
$$

where $w_{21}, w_{22}$ and $\omega_{2}$ are the second row elements of the matrices $\mathcal{W}$ and $\Omega$, respectively. The condition $E_{t} \tilde{\varepsilon}_{c, t+1}=0$ ensures that rational agents do not make systematic mistakes in forecasting the future based on current information. Since $\tilde{\varepsilon}_{c, t}$ can reflect a purely extraneous shock, it can be interpreted as shock to autonomous consumption.

\subsection{Conditions for indeterminacy}

Theorem 1 derives necessary and sufficient conditions for local indeterminacy of the equilibrium path (that is for both eigenvalues of the $\mathcal{W}$ matrix lying inside the unit circle).

Theorem 1 The model equilibrium is locally indeterminate when the following necessary (NC) and "general" (GC) conditions hold:

$$
\begin{aligned}
& \boldsymbol{N C}: \quad \beta<(1+\eta)\left\{\left(1-(1-\alpha-\rho) \frac{\tau_{N}}{1+\tau_{N}}\right)+\rho\left(\frac{1}{\left(1-\tau_{Y}\right)\left(1+s p \tau_{N}\right)}-1\right)\right\} \\
& \boldsymbol{G C}: \max \left\{\frac{1}{\beta(1-\delta)}, \underline{\underline{\mathcal{R}}-1}\right\}<\rho(1+\zeta)+(1-\alpha-\rho)(1+\eta)<\frac{\overline{\mathcal{R}}}{\overline{\mathcal{R}}-1},
\end{aligned}
$$

where $\underline{\mathcal{R}}$ and $\overline{\mathcal{R}}$ are two quantities (defined in the appendix) such that $\underline{\mathcal{R}}>\overline{\mathcal{R}}>1$.

Proof. See Appendix.

To present a neat economic interpretation it is convenient to rewrite the necessary condition in terms of the steady state share of disposable income, and the general conditions in terms of elasticities and cross-elasticities of the demand schedules for capital, regular and underground labor with respect to the three production inputs.

\subsubsection{The Necessary Condition}

The necessary condition suggests that distortionary taxation and tax evasion, combined together, allow to reduce the degree of increasing returns to scale (the parameter $\eta$ ) required for having indeterminacy. ${ }^{7}$ It is here necessary to distinguish between $\tau_{N}$ and $\tau_{Y}$.

Taking $\tau_{Y}$ as given, the higher the probability of being detected evading $p$ (and/or the penalty surcharge factor $s$ ), the more difficult it becomes to allocate resources to the underground sector, and the smaller the quantity $\left(\frac{1}{\left(1-\tau_{Y}\right)\left(1+s p \tau_{N}\right)}-1\right)$ gets. Consider the extreme case where tax evaders are punished with an infinitely large penalty (that is $s \rightarrow \infty$ ). The NC reads: $\beta<$

\footnotetext{
${ }^{7}$ When there is no tax evasion (and therefore no underground labor: when $\tau_{N}=\tau_{Y}=\rho=0$ ) the necessary condition and the general condition reduce to $\beta<(\eta+1)$. The condition for indeterminacy in the standard Farmer and Guo model is $\frac{1}{1-\alpha}<1+\eta$, which is, thus, a necessary and sufficient condition. We would get it by combining together our necessary and general conditions for a no-tax-evasion scenario.
} 
$(1+\eta)\left\{\left(1-(1-\alpha-\rho) \frac{\tau_{N}}{1+\tau_{N}}\right)-\rho\right\}$, suggesting that the parameter region for indeterminacy shrinks when tax evasion becomes extremely costly, and it fails if labor taxes are too high $\left(\tau_{N}>\frac{\beta-(1+\eta)(1-\rho)}{\alpha(1+\eta)-\beta}\right.$, for example). Indeed, when tax evasion is extremely costly/risky, and when taxes are higher than a certain threshold, they "tax away" the externality, in the spirit of Guo and Lansing [10].

The picture is different if we take $\tau_{N}$ as given. An increase in income tax rate $\tau_{Y}$ monotonically increases the quantity $\left(\frac{1}{\left(1-\tau_{Y}\right)\left(1+s p \tau_{N}\right)}-1\right)$, easing, by this hand, the necessary condition. That happens because there is no probability of being detected evading income taxation (on the households' side). Therefore, the higher the income tax rate, the higher would be the underground labor supply; in this sense, resources would be reallocated toward an input that ensures a tax-free externality. In this case we cannot claim that higher income tax rates tax away the externality.

In summary, this analysis suggests that underground economy and tax evasion can be considered as additional economic phenomena inducing local indeterminacy of the equilibrium path, and as an economic rationale for regressive (effective) tax schemes (more details to come in the sequel).

\subsubsection{The General Condition}

The general condition can be re-written in terms of the cross-elasticity of regular (underground) labor demand with respect to underground (regular) labor services $\left(\varepsilon_{\widehat{N}_{U}}^{\widehat{L}_{M}}=\rho(1+\zeta)\right.$ and $\varepsilon_{\widehat{N}_{M}}^{\widehat{L}^{D}}=$ $(1-\alpha-\rho)(1+\eta)$, respectively).

$$
\max \left\{\frac{1}{\beta(1-\delta)}, \underline{\underline{\mathcal{R}}-1}\right\}<\varepsilon_{\widehat{N}_{U}}^{\widehat{L}_{M}^{D}}+\varepsilon_{\widehat{N}_{M}}^{\widehat{N}_{U}^{D}}<\frac{\overline{\mathcal{R}}}{\overline{\mathcal{R}}-1} .
$$

This condition suggests that the labor demand schedules should have a sufficiently large response to changes in equilibrium employment (that is the lower bound inequality $\max \left\{\frac{1}{\beta(1-\delta)}, \frac{\mathcal{\mathcal { R }}}{\underline{\mathcal{R}}-1}\right\}<$ $\varepsilon_{\widehat{N}_{U}}^{\widehat{L}_{M}^{D}}+\varepsilon_{\widehat{N}_{M}}^{\widehat{N}_{U}}$ ), but, at the same time, that this response should not be too large (that is the upper bound inequality $\varepsilon_{\widehat{N}_{U}}^{\widehat{L}_{M}}+\varepsilon_{\widehat{N}_{M}}^{\widehat{N}_{M}}<\frac{\overline{\mathcal{R}}}{\overline{\mathcal{R}}-1}$ ). This condition represents a building block of the theoretical mechanism described below (see Section 3.3).

To better appreciate the economic intuition behind the general condition, then, it is convenient to focus on the lower inequality. It can be rewritten in terms of elasticities of labor demand schedules to changes in capital stock $\varepsilon_{\widehat{K}}^{\widehat{L}_{M}^{D}}=\varepsilon_{\widehat{K}}^{\widehat{L}_{U}^{D}}=\alpha(1+\eta)$; it yields:

$$
\varepsilon_{\widehat{K}}^{\widehat{L}_{M}^{D}} \text { and } \varepsilon_{\widehat{K}}^{\widehat{L}_{U}^{D}}>\frac{s_{I}}{s_{I}+s_{C}}\left\{1+\frac{(1-\delta)(1-\beta)}{\delta}\left(\begin{array}{c}
\widehat{L}_{M}^{D} \\
\widehat{\widehat{N}}_{U}
\end{array}\right.\right.
$$

where $s_{I}$ and $s_{C}$ denote investment and consumption shares, evaluated at the steady state. This condition suggests that regular and underground labor demands should react more to changes in capital stocks rather than changes in labor services, ceteris paribus. In other words, the shifts of labor demands driven by changes in capital stock should be "larger" than those driven by changes in labor services. Again, this result is important for understanding model's mechanism presented below. 


\subsection{The theoretical mechanism}

The theoretical mechanism driving the economy under self-fulfilling beliefs differs from the customary one, and this section describes it by focusing on the labor markets. ${ }^{8}$

Suppose a positive sunspot shock hits the economy. Agents, then, formulate a conjecture on future income and consumption, according to which they believe to be more wealthy. Hence, they would like to consume more and - at the beginning - to work less. The household first order conditions suggest that both labor supply schedules would shift up (from $\widehat{L}_{i}^{S}(0)$ to $\widehat{L}_{i}^{S}(1) i=M, U$ as Figure 1 shows).

Now comes the key difference compared with a standard one-sector model where labor demand slopes upward. In our model both labor demand schedules are sloping down for our parametrization, and therefore the economy would begin plummeting into a recession. ${ }^{9}$ But, in a perfect foresight equilibrium agents are aware that they could reduce their tax burden, increase their disposable income, and therefore could afford the higher conjectured consumption, by allocating resources to the underground input. Notice that this is possible only when the necessary condition is satisfied.

The resource reallocation toward underground labor would then trigger an expansionary mechanism, because it increases returns to capital, regular wage, and therefore equilibrium capital stock and equilibrium regular labor. This in turn, would raise underground labor wage rate, inducing a further expansion in the economy. The general condition ensures that the expansionary shifts of labor demand schedules due to capital stock dominate. This mechanism, eventually, would push the economy into an expansion, which fulfils the initial prophecy of higher consumption (as suggested by labor demands' shift to $\widehat{L}_{i}^{D}(2), i=M, U$ in Figure 1$)$.

Compared to traditional labor market income, the income derived from underground labor activity is subject to a lower expected tax rate when considering both the probability of detection and the evasion penalty. During a belief-driven expansion, the household allocates more time to both traditional and underground labor supply. In equilibrium, this action serves to lower the effective labor tax rate faced by the household, thus providing stimulus to aggregate labor supply so as to make the initial expansion self-fulfilling. The mechanism here is similar to a "regressive tax" (i.e. Guo and Lansing, [10]); the household's effective tax rate depends negatively on the level of total labor income. To conclude, an underground sector and the associated tax evasion offers an economic rationale for a regressive tax rate.

\footnotetext{
${ }^{8}$ In a discrete time model like this, however, everything happens simultaneously, and the following description has just an explanatory purpose.

${ }^{9}$ When labor demand schedule is upward sloping, as in the benchmark one sector model with indeterminacy, an upward shift of labor supply schedules increases equilibrium labor services; this supports an increase in income flow, and generates an expansion. This would be consistent with the households' conjectures, and would lead the economy into a self-fulfilled expansion.
} 

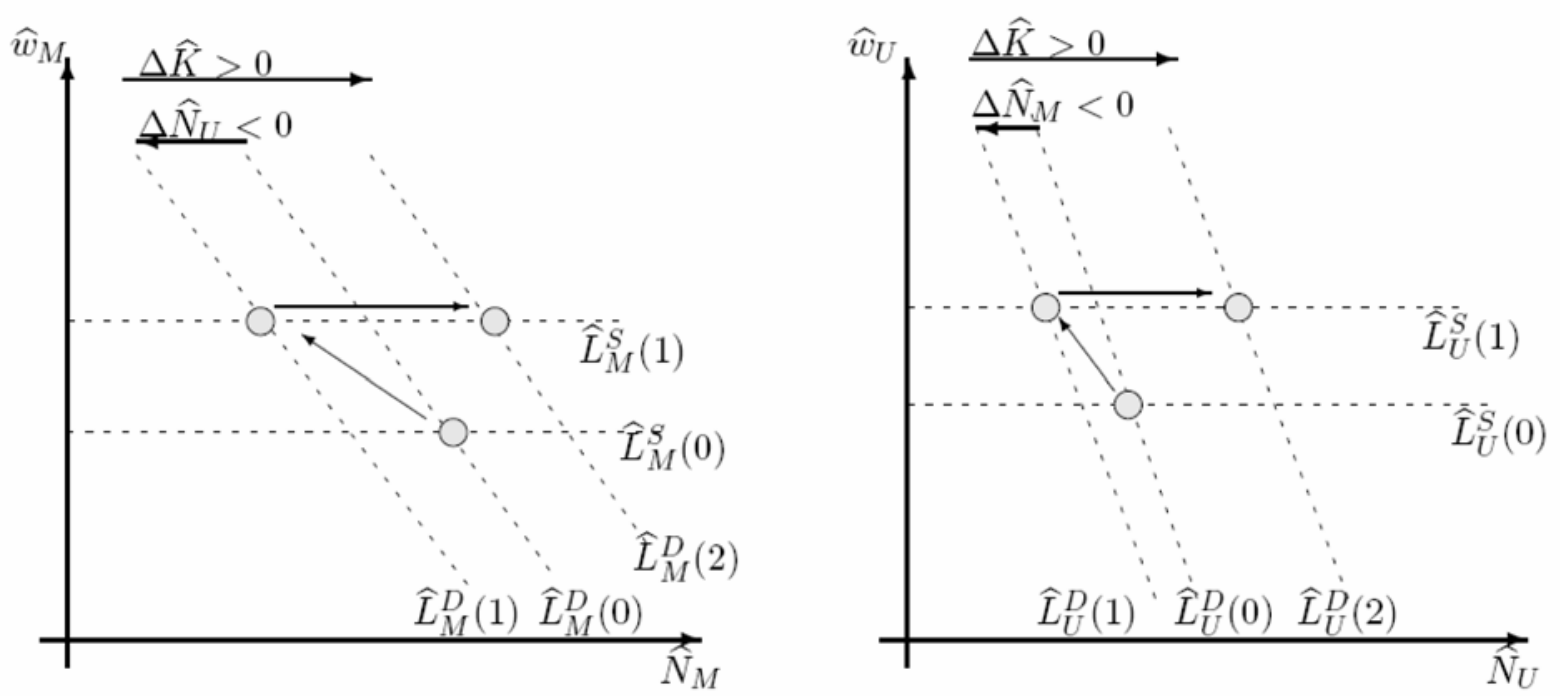

Figure 1: Theoretical Mechanism. Regular $\left(\hat{L}_{M}^{S}\right)$ and underground $\left(\hat{L}_{U}^{S}\right)$ labor supply schedules shift upward (to $\hat{L}_{M, U}^{S}(1)$ ) after a positive sunspot shock; the economy would enter into a recession $\left(\Delta \hat{N}_{M}<0\right.$ and $\left.\Delta \hat{N}_{U}<0\right)$, as labor demands $\hat{L}_{M}^{D}(0)$ and $\hat{L}_{U}^{D}(0)$ are negatively sloped. The crossinteraction between labor markets would further strengthen the inward shifts of labor demands. But, in a perfect foresight equilibrium, the labor input reallocation toward underground labor input would increase households' disposable income, who can consume and invest more. This triggers the capital accumulation $(\Delta \hat{K}>0)$ that shifts out both labor demand schedules to $\hat{L}_{M}^{S}(2)$ and $\hat{L}_{U}^{S}(2)$.

\section{Numerical results}

The introduction of an underground economy ensures that labor demand schedules are well behaved (in the sense that they slope down in the wage-employment plane). To have a complete understanding of the model, we then calibrate it for the United States economy and compare the impulse response functions to a positive sunspot shock with those generated with the benchmark one sector model. Finally a sensitivity analysis exercise shows the results' robustness and underlines that even a tiny underground sector (in terms of GDP percentage points) matters for inducing local indeterminacy of the equilibrium.

\subsection{Well behaved labor demand schedules}

In this model both labor demand schedules are well behaved (in the sense that they slope down), compared to standard one-sector economy models where the necessary and general condition for indeterminacy requires that labor demand is upward sloping. Just observe that in our model $\frac{\partial \widehat{L}_{M}^{D}}{\partial \widehat{N}_{M}}=$ $(1+\eta)(1-\alpha-\rho)-1<0$ and $\frac{\partial \widehat{L}_{U}^{D}}{\partial \widehat{N}_{U}}=(1+\zeta) \rho-1<0$ are both negative, as long as the following condition holds: 
Condition 1 Linearized labor demand schedules slope in the planes $\left(\widehat{N}_{M}, \widehat{w}_{M}\right)$ and $\left(\widehat{N}_{U}, \widehat{w}_{U}\right)$ down if $\eta^{*}<\frac{\alpha+\rho}{1-\alpha-\rho}$ and $\zeta^{*}<\frac{1-\rho}{\rho}$.

Condition 1 is satisfied for our parameterizations (see Table II): for $\alpha=0.23$ and $\rho=0.088$, the regular externality parameter should be less than $\eta^{*}=0.46$ and the underground specific externality parameter should be less than $\zeta^{*}=10.36{ }^{10}$ Notice that in a model with no underground labor input (that is for $\rho=0$ ), this condition would be much more restrictive and not compatible with the stationary state being indeterminate. Indeed, for $\rho=0$ the regular labor demand schedule slopes down if $\eta^{*}<0.2987$, and for this figure the model stationary state is saddle path stable. Hence the introduction of underground labor input eases the conditions for having a sloping down labor demand schedule. A similar argument applies for the underground labor demand schedule.

As expected, the explicit introduction of distortionary taxation combined with these phenomena into a one-sector general equilibrium model allows to reduce the aggregate degree of increasing returns required for indeterminacy, and for having well behaved demand schedules for production inputs (in the sense that they slope down).

\subsection{Parametrization and impulse response functions}

Parametrization. The model is parameterized for the United States economy; calibration is based on seasonally adjusted series from 1970 to 2001, expressed in constant 1995 prices. Actual data for the United States economy are drawn from Farmer [8]. The system of equations we use to compute the dynamic equilibria of the model depends on a set of 12 parameters. Three pertain to household preferences, $\left(B_{0}, B_{1}, \beta\right)$, four to the institutional context (the probability of a firm being detected evading $p$, and the surcharge factor $s$, the two tax rates $\tau_{Y}$ and $\tau_{N}$ ), and the remaining five parameters to technology (the factor shares $\alpha, \rho$, the capital depreciation rate $\delta$, the "regular" externality parameter $\eta$, and the one associated to the underground labor $\zeta$ ). A starred parameter denotes the precise calibrated value. Table I below includes calibrated values of all parameters.

\section{Table I}

The calibration of parameters that are more closely related to tax evasion and the underground economy deserves more attention. For the probability of being detected $p$, we rely on Joulfaian and Ride [14], which estimate that the probability of auditing in the United States ranges between 4.6 percent and 5.7 percent. We choose the higher value, $p^{*}=0.057$, but results do not significantly change if we consider the lower value 4.7 percent.

To stop tax evasion schemes, the Internal Revenue Service has recently undertaken a nationally coordinated strategy to address abusive tax evasion schemes (see e.g. Internal Revenue Service Public Announcement Notice 97-24). Violations of the Internal Revenue Code may result in civil penalties,

\footnotetext{
${ }^{10}$ This result is robust to a large set of parameters, as long as the regular externality $\eta$ is sufficiently small. The quantity $(1-\alpha-\rho)$ is in fact positive and small. If $\eta$ gets "too big", the slope of the regular labor demand schedule $(1+\eta)(1-\alpha-\rho)-1$ becomes positive.
} 
which includes a fraud penalty of up to 75 percent of the underpayment of tax attributable to the fraud in addition to the taxes owed. Therefore we set the surcharge factor $s^{*}=1.75$.

The disutility parameters in the utility function $B_{0}^{*}$ and $B_{1}^{*}$ are set to calibrate the steady state values of the equilibrium regular labor services to $N_{M}^{\star}=0.32$ and the steady state value of underground labor share to $\frac{N_{U}^{\star}}{N_{M}^{\star}}=0.10$, respectively. This is equivalent to imposing that the underground labor services equal the 8.8 percent of aggregate labor services, as the data suggest.

The calibration of the externality parameters $\eta$ and $\zeta$ deserves little more attention. They are calibrated by using the regular labor demand schedule and the aggregate production function. More precisely, rewrite these two equations in terms of the empirically-known macroeconomic ratios $\frac{K}{N_{M}}$ , $\frac{N_{U}}{N_{M}}$, take a logarithmic transformation, and then solve for $\eta$ and $\zeta$. We obtain $\eta^{*}=0.44$ and $\zeta^{*}=0.28 .{ }^{11}$ Table II compares two alternative parameterizations with the benchmark model (Farmer and Guo [7]) and with actual data.

\section{Table II}

Section 5 shows that the degree of increasing returns can be lowered even more. A plausiblycalibrated underground sector can reduce the required degree on increasing returns for indeterminacy to around 1.4, as compared to a value of about 1.6 in a model without underground labor. Although this is not the main goal of the paper, we consider it as a welcome additional result.

Impulse response functions. Figure 3 compares our model's impulse response functions (IRFs) (solid lines) with those generated by a Farmer and Guo [7]-type of the model, which we consider our benchmark (dashed lines). Following the literature (e.g. Farmer and Guo [7]), we pick the standard deviation of sunspot that match the aggregate fluctuations. A sunspot shock increases consumption, equilibrium total employment and production output, all dynamic responses follow a non-monotonic pattern and revert back to the stationary state.

The qualitative response patterns of aggregate quantities are comparable with those of the benchmark one sector model, as the Figure suggests. ${ }^{12}$ Notice, however, that the mechanism underlying a class of model with labor heterogeneity (i.e. regular and underground labor) is completely different from the customary one, as Section 3.3 suggests. In addition, a distinctive characteristic of our model is the much stronger responses of capital interest rate $(r)$ and aggregate labor productivity $(w)$. This is the consequence of the relatively more efficient propagation mechanism operating in our model.

\footnotetext{
${ }^{11}$ More precisely, rewrite the regular labor demand schedule and the aggregate production function in terms of $\frac{K}{N_{M}}$ , $\frac{N_{U}}{N_{M}}$, take a logarithmic transformation, and then solve for $\eta$ and $\zeta$. The final expressions read:$$
\eta^{*}=\frac{-\ln \frac{(1-\alpha-\rho)}{1+\tau N}-\alpha \ln \left(\frac{K}{N_{M}}\right)-\rho \ln \left(\frac{N_{U}}{N_{M}}\right)+\ln w_{M}}{\left(\alpha \ln \left(\frac{K}{N_{M}}\right)+\ln N_{M}+\rho \ln \left(\frac{N_{U}}{N_{M}}\right)\right)} \text { and } \zeta^{*}=\frac{\ln Y-\alpha\left(1+\eta^{*}\right) \ln \left(\frac{K}{N_{M}}\right)-\rho \ln \left(\frac{N_{U}}{N_{M}}\right)-\left(1+\eta^{*}\right) \ln N_{M}}{\rho \ln \left(\frac{N_{U}}{N_{M}}\right)}
$$

Data Source: $Y$ : NIPA Table 1.4.1 (revised Feb 2004); $N_{M}$ : NIPA Table 6.4B (revised Feb 2004); GDP Deflator: NIPA Table 1.5.1 (revised Feb 2004); Capital labor ratio $\frac{K}{N_{M}}$ is taken from Ghosal ([9]) estimates (precisely, we use the mean value of the estimated distribution for the United States manufacturing sector, which equals 31.56).

${ }^{12}$ Such a comparison would be reasonable, because the official GDP estimates for the United States incorporate an estimate of underground sector contribution.
} 

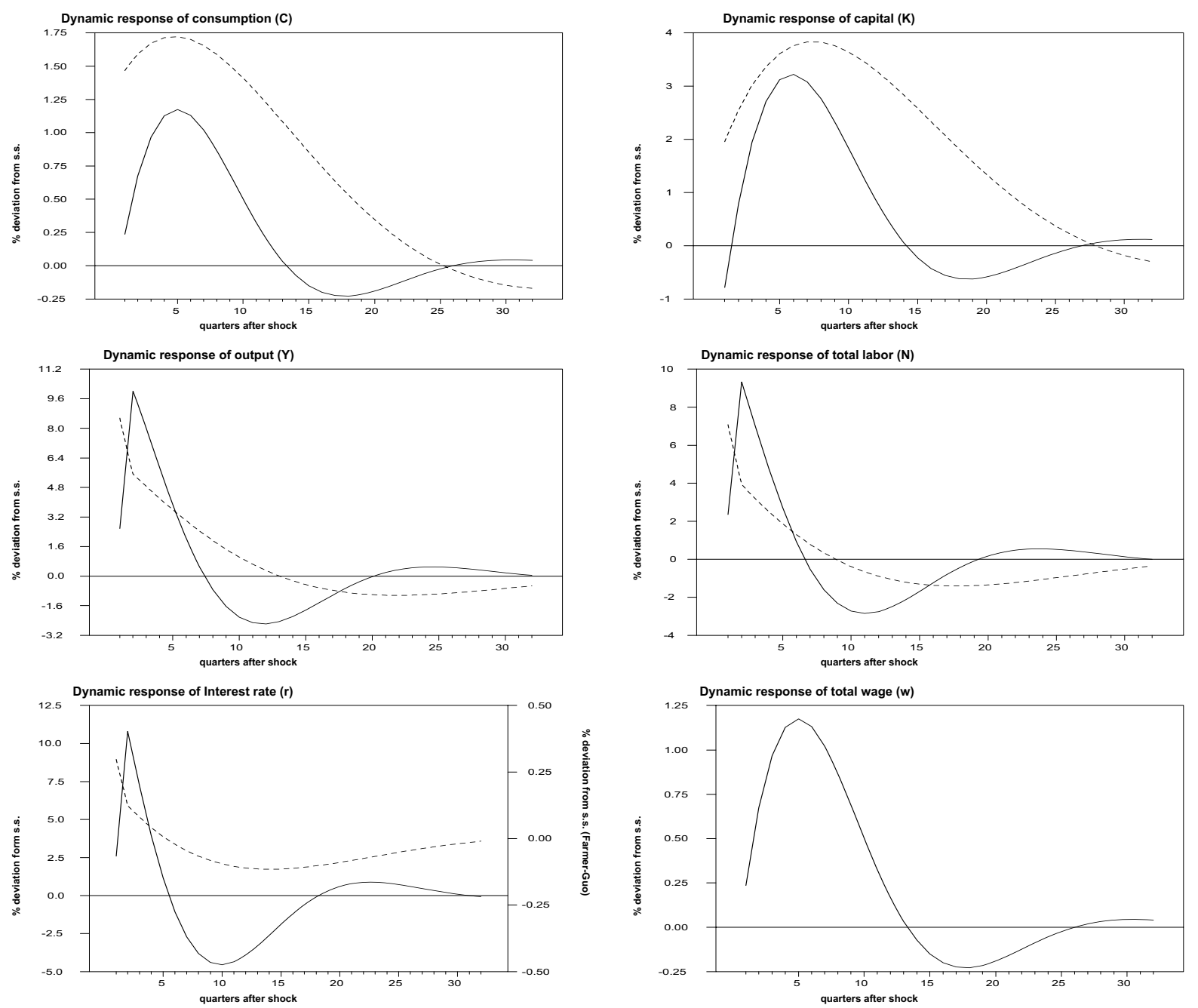

Figure 2: Impulse Response Functions. The figure shows the first 32 quarter response of main endogenous variables to a positive sunspot shock, for the baseline parameterization in Table II $(\eta=0.44 ; \zeta=0.28)$. The response patterns would not be qualitatively different for the other parameterizations under which the stationary state is indeterminate. Notice that the response functions in the bottom-left panel are plotted in dual scale. The curves are the quarterly percentage deviations from a baseline scenario where there is no uncertainty. 
In other words, the model predicts that a positive sunspot shock under indeterminacy should make investment more appealing (in order to self-fulfil the expansionary expectations). A natural way to verify this issue is to rewrite the Euler equation, isolating the covariance term between marginal utility of consumption and investment returns $\operatorname{Cov}\left(C_{t+1}^{-1}, R_{t+1}\right)$, i.e.

$$
E_{t}\left(C_{t}^{-1}\right)=\beta E_{t}\left(C_{t+1}^{-1} R_{t+1}\right) \Rightarrow E_{t}\left(C_{t}^{-1}\right)=\beta E_{t}\left(C_{t+1}^{-1}\right) E_{t}\left(R_{t+1}\right)+\beta \operatorname{Cov}\left(C_{t+1}^{-1}, R_{t+1}\right)
$$

Now, investment becomes more appealing when the returns to saving are high in times when marginal utility of consumption is low. Hence, the lower the covariance $\operatorname{Cov}\left(C_{t+1}^{-1}, R_{t+1}\right)$, the more appealing investment is. We should expect, therefore, that the stronger the self-fulfilling prophecies, the higher the correlation between consumption and returns. A numerical exercise confirms this claim. When self-fulfilling prophecies are low (degree of returns to scale equal to 1.37) then $\operatorname{Cov}\left(C_{t+1}^{-1}, R_{t+1}\right)=0.63$; consistently with our claim, when self-fulfilling prophecies get stronger (degree of returns to scale equals to 1.41) then $\operatorname{Cov}\left(C_{t+1}^{-1}, R_{t+1}\right)$ monotonically decreases to 0.19.

\section{Sensitivity Analysis}

For the baseline parametrization (Section 4.2, Table II), the model's attractor is a sink; the matrix $\mathcal{W}$ of the linearized system $E_{t} \widehat{S}_{t+1}=\mathcal{W} \widehat{S}_{t}+\Omega \mathcal{E}_{t+1}$ (previously defined) has two complex conjugated eigenvalues: the two roots equal $0.8297+0.2185 i$ and $0.8297-0.2185 i$. The sensitivity analysis in the sequel restricts its attention to the externality parameters $\eta$ and $\zeta$. Table III shows that the value of $\zeta$ can be significantly lowered and that of $\eta$ increased with a little subsequent increase in the degree of returns to scale - which, however, remains below the benchmark one-sector model. In this sense the Table suggests that there exists a trade-off between $\zeta$ and $\eta$.

\section{Table III}

The last four rows of the table are particularly instructive for understanding the models' mechanism. They consider four extremes cases: two in which the underground sector does not produce any external effect $\left(\eta^{*}=0.44 ; \zeta^{*}=0.00\right.$ and $\left.\eta^{*}=0.40 ; \zeta^{*}=0.00\right)$, and two in which the regular sector has no external effect $\left(\eta^{*}=0.00 ; \zeta^{*}=0.28\right.$ and $\left.\eta^{*}=0.00 ; \zeta^{*}=0.56\right)$.

Now, the equilibrium path is still locally indeterminate even when the underground sector does not contribute to the externality effect at all, i.e. when $\zeta$ is equal to zero. This suggests that the very existence of the underground sector is the key for the theoretical mechanism of this model to be operating. Indeed, both labor demand schedules are still well behaved when the undergroundspecific external effect is zero, because the elasticity of the log-linearized regular demand schedule $((1+\eta)(1-\alpha-\rho)-1)$ does not depend on the underground external effect $\zeta$. Intuitively speaking, that happens, because the underground labor share $\rho$ "absorbes" some of the regular sector external effect. On the other hand, if the underground sector labor share were getting smaller and smaller zero $(\rho \rightarrow 0)$, the regular labor demand would slope up again, and the mechanism would fail. 
In addition, the dynamical system characterizing the model becomes saddle path stable when the regular external effect $\eta$ is set to zero. This result is consistent with the model and with the economic intuition. Regular labor services are the largest, in terms of income share, production factor, and its external effect has to be crucial for local indeterminacy.

Finally, the Table suggests that the model still presents indeterminacy for an even smaller (as a percentage of the GDP) underground economy sector $\left(\left(\frac{N_{U}}{N}\right)^{*}\right.$ lowered from 0.088 to 0.04$)$.

\section{Conclusions}

This paper proposes a one-sector dynamic general equilibrium model augmented with tax evasion and underground activities; it includes an additional type of labor services along with the standard capital and regular labor, and a proper taxation structure. The model displays increasing returns to scale due to externalities in both regular and underground inputs, capable of inducing local indeterminacy of the equilibrium path.

The theoretical mechanism driving such a model departs from the customary one. It turns out that prophecies of a higher expected income are self-fulfilled through a resource reallocation toward the underground labor services, which allows to evade taxes, and therefore to have additional resources (the tax wedge) for satisfying the higher desired consumption profile. It is important to stress that the explicit introduction of these phenomena into a one-sector general equilibrium model allows to have well behaved demand schedules for production inputs (in the sense that they slope down), while a traditional one-sector models is characterized by upward sloping labor demand schedule. 


\section{References}

[1] C. Bajada, Estimates of the Underground Economy in Australia, Economic Record, 75 (1999), 369-84.

[2] S. Basu, J. Fernald, Returns to Scale in U.S. Production: Estimates and Implications, Journal of Political Economy, 105 (1997), 249-83.

[3] J. Benhabib, R. Farmer, Indeterminacy and Increasing Returns, Journal of Economic Theory, 63 (1994), 19-41.

[4] J. Benhabib, R. Farmer, Indeterminacy and Sector Specific Externalities, Journal of Monetary Economics 371 (1996), 421-443.

[5] F. Busato, B. Chiarini, Market and underground activities in a two-sector dynamic equilibrium model, Economic Theory, 23 (2004), 831-861.

[6] A. Cowell, Cheating the Government The Economics of Evasion, MIT Press, Cambridge, 1990.

[7] R. Farmer, J. Guo, Real Business Cycle and the Animal Spirit Hypothesis, Journal of Economic Theory, 63 (1994), 42-72.

[8] R. Farmer, The macroeconomics of self-fulfilling prophecies, MIT Press, Cambridge, 1999.

[9] V. Ghosal, Demand Uncertainty and the Capital-Labor Ratio: Evidence from the U.S. Manifacturing Sector, Review of Economics and Statistics, 73 (1991), 157-161.

[10] J. Guo, K. Lansing, Indeterminacy and Stabilization Policy, Journal of Economic Theory, 82 (1998), 481-490.

[11] J. Guo, K. Lansing, Maintenance Labor and Indeterminacy under Increasing Returns to Scale, unpublished manuscript, University of California, Riverside, (2004).

[12] G. Gandolfo, Economic Dynamics, $2^{\text {nd }}$ edition, Springer, Berlin, 1998.

[13] M. Jimenez, D. Marchetti, Interpreting the procyclical productivity of manufacturing sectors: can we really rule out external effects?, Applied Economics, 34 (2002), 805-817.

[14] D. Joulfaian, M. Rider, Differential Taxation and Tax Evasion by Small Business, National Tax Journal, 4, (1998), 675-687.

[15] T. Paglin, The Underground Economy: New Estimates from Household Income and Expenditure Surveys, Yale Law Journal, 103 (1994), 2239-57.

[16] R. Perli, Indeterminacy, Home Production and the Business Cycle: a Calibrated Analysis Journal of Monetary Economics, 41 (1998), 105-125.

[17] A. Sbordone, Interpreting the procyclical productivity of manufacturing sectors: external effects or labour hoarding, Journal of Money, Credit and Banking, 29 (1997), 26-45.

[18] F. Schneider, D. Enste, Shadow Economies: Size, Causes and Consequences, Journal of Economic Literature, 38 (2000), 77-114.

[19] V. Tanzi, The Underground Economy in the United States: Estimates and Implications, Banca Nazionale del Lavoro Quarterly Review, 135 (1980), 427-53.

[20] Y. Wen, Capacity Utilization under Increasing Returns to Scale, Journal of Economic Theory, 81 (1998), 7-36. 
Table I: Parametrization.

\begin{tabular}{l|rr}
\hline \hline Institutional Setting & $p^{*}=0.057$ & $s^{*}=1.75$ \\
Disutility of Working & $B_{0}^{*}=2.50$ & $B_{1}^{*}=2.00$ \\
Preferences & $\beta^{*}=0.984$ & \\
Technology & $\alpha^{*}=0.23$ & $\rho^{*}=0.088 \quad \delta^{*}=0.025$ \\
Effective Tax Rates & $\tau_{N}^{*}=0.153$ & $\tau_{Y}^{*}=0.1186$ \\
\hline
\end{tabular}

Notes: The parameters $\beta^{*}, \alpha^{*}, \delta^{*}$ are from Farmer and Guo [7]; The disutility parameters in the utility function $B_{0}^{*}$ and $B_{1}^{*}$ are set to calibrate the steady state values of the equilibrium regular labor services to $N_{M}^{\star}=0.32$ and the steady state value of underground labor share to $\frac{N_{U}^{\star}}{N_{M}^{\star}}=0.10$ respectively; this is equivalent to imposing that the underground labor services equals the 8.8 percent of aggregate labor services, as the data suggest (see Schneider and Enste [18]); $p^{*}$ and $s^{*}$ are from Joulfaian and Ride [14]; the effective income tax rate $\tau_{Y}^{*}$ is computed from the "Effective Tax Rates, 1979-1997", Table H-1a, prepared by the Congressional Budget Office; social security tax rate is taken from www.socialsecurity.com; we choose the value applying for the 1990s and later, which equals $\tau_{N}^{*}=0.153$ (http://www.ssa.gov/OACT/ProgData/taxRates.html). 
Table II: Externality Parameters.

\begin{tabular}{l|ccc} 
& $\eta^{*}$ & $\zeta^{*}$ & $\frac{\text { Aggregate degree of }}{\text { increasing returns to scale }}$ \\
\hline \hline Baseline Parametrization & 0.44 & 0.28 & 1.43 \\
Alternative Parametrization & 0.39 & 0.28 & 1.38 \\
& & & \\
Benchmark (Farmer and Guo [7]) & 0.60 & - & 1.60 \\
Actual Data (Farmer and Guo [7]) & & & $\leq 1.20$ \\
\hline
\end{tabular}

Notes: $\eta^{*}$ : calibrated regular externality parameter; $\zeta^{*}:$ calibrated underground externality parameter; overall degree of returns to scale reads: $(1+\eta)(1-\rho)+(1+\zeta) \rho$. 
Table III: Sensitivity Analysis.

\begin{tabular}{rrr|rrr}
\hline$\left(\frac{N_{U}}{N}\right)^{*}$ & $\eta^{*}$ & \multicolumn{1}{c}{$\zeta$} & eigenvalues & $\frac{\text { Attractor }}{\text { topological properties }}$ & $\frac{\text { Aggregate degree of }}{\text { increasing returns to scale }}$ \\
\hline \hline 0.088 & 0.29 & 1.50 & $0.9629 \pm 0.2428 i$ & sink & 1.396 \\
0.088 & 0.29 & 1.30 & $0.9292 \pm 0.2912 i$ & sink & 1.378 \\
0.088 & 0.29 & 1.00 & $0.7115 \pm 0.4421 i$ & sink & 1.352 \\
0.088 & 0.29 & 0.90 & $-0.01 ; 0.19$ & stable node & 1.343 \\
0.088 & 0.29 & 0.70 & $0.7 ; 2.0$ & saddle & 1.326 \\
0.088 & 0.45 & 0.28 & $0.8437 \pm 0.2097 i$ & sink & 1.435 \\
0.088 & 0.4 & 0.28 & $0.666 \pm 0.2263 i$ & sink & 1.389 \\
0.088 & 0.39 & 0.28 & $0.5509+0.1487 i$ & sink & 1.38 \\
0.088 & 0.38 & 0.28 & $-0.06 ; 0.66$ & stable node & 1.371 \\
0.088 & 0.3 & 0.28 & $0.8 ; 1.5$ & saddle & 1.298 \\
0.040 & 0.40 & 1.00 & $0.8911 \pm 0.2278 i$ & sink & 1.424 \\
0.040 & 0.35 & 1.20 & $0.8238 \pm 0.3020 i$ & sink & 1.384 \\
0.040 & 0.30 & 1.50 & $0.3481 \pm 0.3781 i$ & sink & 1.348 \\
0.040 & 0.29 & 1.50 & $0.56 ; 15.1$ & saddle & 1.338 \\
0.088 & 0.44 & 0.00 & $0.6304 \pm 0.1254 i$ & sink & 1.401 \\
0.040 & 0.40 & 0.00 & $0.5833 \pm 0.1801 i$ & sink & 1.384 \\
0.088 & 0.00 & 0.28 & $1.412 ; 0.874$ & saddle & 1.025 \\
0.040 & 0.00 & 0.56 & $1.426 ; 0.866$ & saddle & 1.049 \\
\hline
\end{tabular}

Notes: $\left(\frac{N_{U}}{N}\right)^{*}$ : underground labor share; $\eta$ : regular externality parameter; $\zeta$ : underground-specific externality parameter. Labor demand schedules are well behaved (sloping down) for all parameterizations included in the table. 


\section{Appendix}

\section{Proof of Proposition 1.}

Evaluating the firms' and households' first order conditions at the stationary state yield:

$$
\begin{aligned}
B_{0} & =C^{-1}\left(1-\tau_{Y}\right) w_{M} \\
C^{-1} w_{U} & =B_{0}+B_{1} \\
r & =\frac{\beta^{-1}-1+\delta}{1-\tau_{Y}} \\
C & =\left(1-\tau_{Y}\right)\left(w_{M} N_{M}+r_{K}\right)+w_{U} N_{U}-\delta K \\
\tilde{w}_{M} & =\left(1+\tau_{N}\right) w_{M}=(1-\alpha-\rho) K^{\alpha(1+\eta)} N_{M}^{(1-\alpha-\rho)(1+\eta)-1} N_{U}^{\rho(1+\zeta)} \\
\tilde{w}_{U} & =\left(1+\tilde{\tau}_{N}\right) w_{U}=\rho K^{\alpha(1+\eta)} N_{M}^{(1-\alpha-\rho)(1+\eta)} N^{\rho(1+\zeta)-1}
\end{aligned}
$$

where $\tilde{\tau}_{N}=s p \tau_{N}$. Rewrite the Euler Equation $r=\left(\beta^{-1}-1+\delta\right) /\left(1-\tau_{Y}\right)$ as

$\frac{\beta-1-1+\delta}{1-\tau Y}=\alpha K^{\alpha(1+\eta)-1} N_{M}^{(1-\alpha-\rho)(1+\eta)} N_{U}^{\rho(1+\zeta)}=\alpha K^{\alpha(1+\eta)-1} N_{M}^{(1-\alpha)(1+\eta)}\left(\frac{N_{U}}{N_{M}}\right)^{\rho(1+\zeta)}$

where the quantity $\left(\frac{N_{U}}{N_{M}}\right)^{\rho} \frac{N_{U}^{\rho \zeta}}{N_{M}^{\rho j}}$ is approximated as $\left(\frac{N_{U}}{N_{M}}\right)^{\rho} \frac{N_{U}^{\rho \zeta}}{N_{M}^{\rho j}}$

Claim 1 Ratio $\frac{N_{U}}{N_{M}}$ is stationary.

Proof. Combining $B_{0}=C^{-1}\left(1-\tau_{Y}\right) w_{M}$ with $C^{-1} w_{U}=B_{0}+B_{1}$, yields

$$
\frac{B_{0}}{\left(B_{0}+B_{1}\right)\left(1-\tau_{Y}\right)}\left(\frac{\rho}{1-\alpha-\rho}\right)\left(\frac{1+\tau_{N}}{1+\tilde{\tau}_{N}}\right)=\left(\frac{N_{U}}{N_{M}}\right)^{\star}
$$

Notice that the stationary labor ratio $\left(\frac{N_{U}}{N_{M}}\right)^{\star}$ is independent of any input-specific externality.

Claim 2 The quantity $K^{\alpha(1+\eta)-1} N_{M}^{(1-\alpha)(1+\eta)}$ is stationary.

Proof. Combining (1) with (2), we obtain:

$$
\frac{\beta-1-1+\delta}{(1-\tau Y) \alpha}\left[\frac{B_{0}}{\left(B_{0}+B_{1}\right)\left(1-\tau_{Y}\right)}\left(\frac{\rho}{1-\alpha-\rho}\right)\left(\frac{1+\tau_{N}}{1+\tilde{\tau}_{N}}\right)\right]^{-\rho(1+\zeta)}=K^{\alpha(1+\eta)-1} N_{M}^{(1-\alpha)(1+\eta)}
$$

which establishes our claim, since the left-hand side is constant.

Claim 3 There exists a unique stationary equilibrium for aggregate labor supply $N_{M}^{\star}>0$

Proof. Consider the feasibility constraint:

$$
C=K\left(\left(1-\tau_{Y}\right)\left(\frac{w_{M} N_{M}}{K}+r\right)+\frac{w_{U} N_{U}}{K}-\delta\right)
$$


and observe that the quantities $\frac{w_{M} N_{M}}{K}$ and $\frac{w_{U} N_{U}}{K}$ are stationary, too:

$$
\begin{aligned}
\frac{w_{M} N_{M}}{K} & =\frac{1-\alpha-\rho}{1+\tau_{N}}\left(\frac{\beta^{-1}-1+\delta}{\left(1-\tau_{Y}\right) \alpha}\right)=\bar{\Psi}_{M} \\
\frac{w_{U} N_{U}}{K} & =\frac{\rho}{1+\tilde{\tau}_{N}}\left(\frac{\beta^{-1}-1+\delta}{\left(1-\tau_{Y}\right) \alpha}\right)=\bar{\Psi}_{U}
\end{aligned}
$$

Substituting then (4), (5) into feasibility constraint (3), yields:

$$
C=K\left(\left(1-\tau_{Y}\right)\left(\bar{\Psi}_{M}+\frac{\beta^{-1}-1+\delta}{1-\tau_{Y}}\right)+\bar{\Psi}_{U}-\delta\right)
$$

To derive the stationary equilibrium for total labor supply, substitute (6) into $B_{0}=C^{-1}\left(1-\tau_{Y}\right) w_{M}$ to obtain:

$$
\frac{B_{0}\left(\left(1-\tau_{Y}\right)\left(\bar{\Psi}_{M}+\frac{\beta^{-1}-1+\delta}{1-\tau_{Y}}\right)+\bar{\Psi}_{U}-\delta\right)}{\left(1-\tau_{Y}\right)(1-\alpha-\rho)}=K^{\alpha(1+\eta)-1} N_{M}^{(1-\alpha)(1+\eta)}\left(\frac{N_{U}}{N_{M}}\right)^{\rho(1+\zeta)} N_{M}^{-1} .
$$

Now, since $K^{\alpha(1+\eta)-1} N_{M}^{(1-\alpha)(1+\eta)}$ and $\frac{N_{U}}{N_{M}}$ are constant, the above equation gives the stationary value of $N_{M}^{\star}$ in proposition 1 .

The final step is to compute the stationary equilibrium value for $K^{\star}$. Combining (3) with the value of $N_{M}^{\star}$ it turns out that $K^{\star}$ is stationary, and it reads

$$
K^{\star} \simeq\left(\frac{\alpha\left(1-\tau_{Y}\right)}{\beta^{-1}-1+\delta}\right)^{\frac{1}{1-\alpha(1+\eta)}}\left(\frac{1+\tau_{N}}{1+\tilde{\tau}_{N}} \frac{B_{0}}{\left(B_{0}+B_{1}\right)\left(1-\tau_{Y}\right)} \frac{\rho}{1-\alpha-\rho}\right)^{\frac{\rho(1+\zeta)}{1-\alpha(1+\eta)}}\left(N_{M}^{\star}\right)^{\frac{(1-\alpha)(1+\eta)}{1-\alpha(1+\eta)}}
$$

Proof of Theorem 1. Preliminaries. The proof of theorem 1 develops into 3 different steps: i) convert the linearized equation for the symmetric equilibrium into a into a $2 \times 2$ dynamic system in the variables $\widehat{K}_{t}$ and $\widehat{C}_{t}$; ii) determine the necessary and sufficient condition, relative to the system's coefficients matrix, for local stability (i.e. indeterminacy of equilibrium paths); iii) show that such conditions lead to NC and GC.

Step i): We first consider the equilibrium in the two labor markets, with the corresponding labor demand and supply functions:

$$
\begin{array}{rll}
\text { demand for } N^{M}: & \widehat{w}_{t}^{M}=[(1+\eta) \alpha] \widehat{K}_{t}+[(1+\eta)(1-\alpha-\rho)-1] \widehat{N}_{t}^{M}+[(1+\zeta) \rho] \widehat{N}_{t}^{U} \\
\text { demand for } N^{U}: & \widehat{w}_{t}^{U}=[(1+\eta) \alpha] \widehat{K}_{t}+[(1+\eta)(1-\alpha-\rho)] \widehat{N}_{t}^{M}+[(1+\zeta) \rho-1] \widehat{N}_{t}^{U} \\
\text { supply for } N^{M}: & \widehat{w}_{t}^{M}=\widehat{C}_{t} \\
\text { supply for } N^{U}: & \widehat{w}_{t}^{U}=\widehat{C}_{t}
\end{array}
$$

As it is $\widehat{w}_{t}^{M}=\widehat{w}_{t}^{U}$, from the two demand functions we have: $\widehat{N}_{t}^{U}=\widehat{N}_{t}^{M}$. Thus the demand for labor $\widehat{N}_{t}^{M}$ turns out to be:

$$
\widehat{N}_{t}^{M}=\widehat{N}_{t}^{U}=-\left(\frac{1}{\varphi_{2}}\right) \widehat{C}_{t}+\left[(1+\eta) \alpha \frac{1}{\varphi_{2}}\right] \widehat{K}_{t}
$$

where $\varphi_{2}$ is equal to:

$$
\varphi_{2}=1-(1+\eta)(1-\alpha-\rho)-(1+\zeta) \rho
$$


Now it is possible to deal with the Euler equation: $\widehat{C}_{t}=\widehat{C}_{t+1}-[1-\beta(1-\delta)] \widehat{r}_{t+1}$. Consider the demand for capital: $\widehat{r}_{t}=[\alpha(1+\eta)-1] \widehat{K}_{t}+[(1+\eta)(1-\alpha-\rho)] \widehat{N}_{t}^{M}+[(1+\zeta) \rho] \widehat{N}_{t}^{U}$, and substitute them in the Euler equation:

$$
\widehat{C}_{t}=\widehat{C}_{t+1}-[1-\beta(1-\delta)]\left\{[\alpha(1+\eta)-1] \widehat{K}_{t+1}+[(1+\eta)(1-\alpha-\rho)] \widehat{N}_{t+1}^{M}+[(1+\zeta) \rho] \widehat{N}_{t+1}^{U}\right\}
$$

By using equation (7), we can write:

$$
\widehat{C}_{t}=\left\{1+[1-\beta(1-\delta)] \frac{\varphi_{1}}{\varphi_{2}}\right\} \widehat{C}_{t+1}-\left\{[1-\beta(1-\delta)]\left[(1+\eta) \alpha\left(1+\frac{\varphi_{1}}{\varphi_{2}}\right)-1\right]\right\} \widehat{K}_{t+1}
$$

or equivalently:

$$
-\left[J_{1}\right] \widehat{K}_{t+1}+\left[J_{2}\right] \widehat{C}_{t+1}=\widehat{C}_{t}
$$

where $\varphi_{1}$ and the $J^{\prime}$ s are functions of the parameters:

$$
\begin{aligned}
\varphi_{1} & =(1+\eta)(1-\alpha-\rho)+(1+\zeta) \rho \\
J_{1} & =[1-\beta(1-\delta)]\left\{\alpha(1+\eta)\left(1+\frac{\varphi_{1}}{\varphi_{2}}\right)-1\right\} ; \\
J_{2} & =\left\{1+[1-\beta(1-\delta)]\left(\frac{\varphi_{1}}{\varphi_{2}}\right)\right\} ;
\end{aligned}
$$

We now pass to the (linearised) budget constraint:

$$
\begin{aligned}
& {\left[1-\tau^{Y}\right] \widehat{y}_{t}-\left[\tau^{N}\left(1-\tau^{Y}\right) \frac{w^{\star M} N^{\star} M}{Y^{\star}}\right]\left(\widehat{w}_{t}^{M}+\widehat{N}_{t}^{M}\right)+} \\
&+\left[\left\{1-\left(1-\tau^{Y}\right)\left(1+s p \tau^{N}\right)\right\} \frac{w^{\star U} N^{\star} U}{Y^{\star}}\right]\left(\widehat{w}_{t}^{U}+\widehat{N}_{t}^{U}\right) \\
&=\left[\frac{C^{\star}}{Y^{\star}}\right] \widehat{C}_{t}+\left[\frac{K^{\star}}{Y^{\star}}\right] \widehat{K}_{t+1}+\left[-(1-\delta) \frac{K^{\star}}{Y^{\star}}\right] \widehat{K}_{t}
\end{aligned}
$$

From the linearized version of labor demands, we know that: $\widehat{w}_{t}^{M}+\widehat{N}_{t}^{M}=\widehat{w}_{t}^{U}+\widehat{N}_{t}^{U}=\widehat{Y}_{t}$; then equation (9) turns out to be:

$$
\left[\frac{K^{\star}}{Y^{\star}}\right] \widehat{K}_{t+1}=[M] \widehat{Y}_{t}-\left[\frac{C^{\star}}{Y^{\star}}\right] \widehat{C}_{t}+\left[(1-\delta) \frac{K^{\star}}{Y^{\star}}\right] \widehat{K}_{t}
$$

where the term:

$$
\begin{aligned}
M= & \left(1-\tau^{Y}\right)-\left(1-\tau^{Y}\right)(1-\alpha-\rho) \frac{\tau^{N}}{\left(1+\tau^{N}\right)}+ \\
& +\rho\left\{1-\left(1-\tau^{Y}\right)\left(1+s p \tau^{N}\right)\right\} \frac{1}{\left(1+s p \tau^{N}\right)}
\end{aligned}
$$

is the share of aggregate income net of taxes.By using the production function $\widehat{Y}_{t}=(1+\eta) \alpha \widehat{K}_{t}+$ $(1+\eta)(1-\alpha-\rho) \widehat{N}_{t}^{M}+(1+\zeta) \rho \widehat{N}_{t}^{U}$ and equations (7), we can rewrite (10) in this way:

$$
\left[\frac{K^{\star}}{Y^{\star}}\right] \widehat{K}_{t+1}=-\left[M\left(\frac{\varphi_{1}}{\varphi_{2}}\right)+\frac{C^{\star}}{Y^{\star}}\right] \widehat{C}_{t}+\left[\alpha(1+\eta) M\left(1+\frac{\varphi_{1}}{\varphi_{2}}\right)+(1-\delta) \frac{K^{\star}}{Y^{\star}}\right] \widehat{K}_{t}
$$


By naming $\frac{C^{\star}}{Y^{\star}}=s_{C}$ and $\frac{K^{\star}}{Y^{\star}}=\frac{I^{\star}}{Y^{\star} \delta}=\frac{s_{I}}{\delta}$, withe the constraint $s_{C}+s_{I}+s_{g}=1$, this equation turns out to be:

$$
\left[s_{I}\right] \widehat{K}_{t+1}=-\left[\delta M\left(\frac{\varphi_{1}}{\varphi_{2}}\right)+\delta s_{C}\right] \widehat{C}_{t}+\left[\delta \alpha(1+\eta) M\left(1+\frac{\varphi_{1}}{\varphi_{2}}\right)+(1-\delta) s_{I}\right] \widehat{K}_{t}
$$

It is interesting to note some relations between the share of aggregate demand on income. From the steady state we have: $r^{\star}=\left(\frac{1}{\beta}-1+\delta\right)\left(1-\tau^{Y}\right)^{-1}$, and from the demand for capital: $\alpha \frac{Y^{\star}}{K^{\star}}=r^{\star}$. By equating for the interest rate $r^{\star}$ and recalling that $\frac{K^{\star}}{Y^{\star}}=\frac{I^{\star}}{Y^{\star} \delta}=\frac{S_{I}}{\delta}$, we obtain:

$$
s_{I}=\frac{\alpha \beta \delta\left(1-\tau^{Y}\right)}{1-\beta(1-\delta)}
$$

From the public budget constraint, we know that:

$$
G^{\star}=\left[\tau^{Y}\right] Y^{\star}+\left[\tau^{N}\left(1-\tau^{Y}\right)\right]\left(w^{\star M} N^{\star M}\right)+\left[s p \tau^{N}-\tau^{Y}\left(1+s p \tau^{N}\right)\right]\left(w^{\star U} N^{\star U}\right)
$$

and by using the demands for inputs, we get:

$$
\begin{aligned}
s_{g}= & \tau^{Y}+\left(1-\tau^{Y}\right)(1-\alpha-\rho) \frac{\tau^{N}}{\left(1+\tau^{N}\right)}+ \\
& +\left\{s p \tau^{N}\left(1-\tau^{Y}\right)-\tau^{Y}\right\} \rho \frac{1}{\left(1+s p \tau^{N}\right)}
\end{aligned}
$$

where $s_{g}=\frac{G^{\star}}{Y \star}$ is the share of fiscal revenues on income. Recalling the expression for $M$, it is easy to check that: $M=1-s_{g}$ and $s_{g}=1-M$. Finally, using the resource constraint: $s_{C}+s_{I}+s_{g}=1$, we can obtain the following relation between the demand shares:

$$
s_{C}=M-s_{I}
$$

Equations (8) and (11) define the equilibrium dynamic system of the model:

$$
\left[\begin{array}{cc}
-J_{1} & J_{2} \\
s_{I} & 0
\end{array}\right]\left[\begin{array}{l}
\widehat{K}_{t+1} \\
\widehat{C}_{t+1}
\end{array}\right]=\left[\begin{array}{cc}
0 & 1 \\
J_{3} & -J_{4}
\end{array}\right]\left[\begin{array}{l}
\widehat{K}_{t} \\
\widehat{C}_{t}
\end{array}\right]
$$

where: $J_{3}=\delta \alpha(1+\eta) M\left(1+\frac{\varphi_{1}}{\varphi_{2}}\right)+(1-\delta) s_{I}$ and $J_{4}=\delta \gamma M\left(\frac{\varphi_{1}}{\varphi_{2}}\right)+\delta s_{C}$. If $J_{2} \neq 0$, i.e. if $\frac{\varphi_{1}}{\varphi_{2}} \neq \frac{1}{1-\beta(1-\delta)}$, the system can be put in ordinary form:

$$
\left[\begin{array}{c}
\widehat{K}_{t+1} \\
\widehat{C}_{t+1}
\end{array}\right]=\left[\begin{array}{cc}
\frac{1}{s_{I}} J_{3} & -\frac{1}{s_{I}} J_{4} \\
\frac{J_{1}}{J_{2} s_{I}} J_{3} & \frac{1}{J_{2}}-\frac{J_{1}}{J_{2} s_{I}} J_{4}
\end{array}\right]\left[\begin{array}{c}
\widehat{K}_{t} \\
\widehat{C}_{t}
\end{array}\right]
$$

where:

$$
\begin{aligned}
& J_{3}=\delta \alpha(1+\eta) M\left(1+\frac{\varphi_{1}}{\varphi_{2}}\right)+(1-\delta) s_{I} \\
& J_{4}=\delta M\left(\frac{\varphi_{1}}{\varphi_{2}}\right)+\delta s_{C}
\end{aligned}
$$

We can directly investigate the condition for indeterminacy. A necessary and sufficient set of 
conditions for a system like the (12) to display stability (see Gandolfo 1998, ch. 5) is the following:

$$
\begin{aligned}
\frac{\left(J_{3}-s_{I}\right)\left(1-J_{2}\right)+J_{1} J_{4}}{s_{I} J_{2}} & >0 \\
\frac{\left(J_{3}+s_{I}\right)\left(1+J_{2}\right)-J_{1} J_{4}}{s_{I} J_{2}} & >0 \\
\frac{s_{I} J_{2}-J_{3}}{s_{I} J_{2}} & >0
\end{aligned}
$$

We can rewrite these conditions in terms of the quantities $\varphi_{1}$ and $\varphi_{2}$ :

Condition 2 (eq.13) It must be: $\frac{\delta\left(M-s_{I}\right)[\beta(1-\delta)-1]\left\{[1-\alpha(1+\eta)]\left(1+\frac{\varphi_{1}}{\varphi_{2}}\right)\right\}}{s_{I}+s_{I}(1-\beta(1-\delta))\left(\frac{\varphi_{1}}{\varphi_{2}}\right)}>0$.

Condition 3 (eq.14) It also must be: $\frac{\left\{\delta\left(M-s_{I}\right)[1-\beta(1-\delta)][1-\alpha(1+\eta)]+2\left[\delta \alpha(1+\eta) M+s_{I}(1-\beta(1-\delta))\right]\right\}\left(\frac{\varphi_{1}}{\varphi_{2}}\right)}{s_{I}+s_{I}(1-\beta(1-\delta))\left(\frac{\varphi_{1}}{\varphi_{2}}\right)}+$ $\frac{\delta\left(M-s_{I}\right)[1-\beta(1-\delta)][1-\alpha(1+\eta)]+2\left[\delta \alpha(1+\eta) M+s_{I}(2-\delta)\right]}{s_{I}+s_{I}(1-\beta(1-\delta))\left(\frac{\varphi_{1}}{\varphi_{2}}\right)}>0$.

Condition 4 (eq.15) Finally, it must hold: $\frac{\left[s_{I}(1-\beta(1-\delta))-\delta M \alpha(1+\eta)\right]\left(\frac{\varphi_{1}}{\varphi_{2}}\right)+\delta\left[s_{I}-M \alpha(1+\eta)\right]}{s_{I}+s_{I}(1-\beta(1-\delta))\left(\frac{\varphi_{1}}{\varphi_{2}}\right)}>0$.

The denominator and the numerators of conditions (2) - (4) can be seen as straight lines in the ratio $\frac{\varphi_{1}}{\varphi_{2}}$, under the assumption that the only changing parameter is $\zeta$. Notice that this parameter appears only in the ratio $\frac{\varphi_{1}}{\varphi_{2}}$. To fix the indeterminacy region in a general way, we need to determine a specific ordering for the four roots ${ }^{13}$ of the l.h.s of conditions (2) - (4):

from eq. (13):

$R_{13}^{0}=-1$

from eq. (14):

$\begin{array}{ll}\text { from eq. (15): } & R_{15}^{0}=-\frac{\delta s_{I}-\delta}{s_{I}(1-\beta(1-\delta))} \\ \text { common denominator: } & R_{D}^{0}=-\frac{1}{1-\beta(1-\delta)}\end{array}$

$R_{14}^{0}=-\frac{\delta\left(M-s_{I}\right)[1-\beta(1-\delta)](1-\alpha(1+\eta))+2\left[\delta \alpha(1+\eta) M+s_{I}(2-\delta)\right]}{\delta\left(M-s_{I}\right)[1-\beta(1-\delta)](1-\alpha(1+\eta))+2\left[\delta \alpha(1+\eta) M+s_{I}(1-\beta(1-\delta))\right]}$

We can now state the following:

Theorem 2 (Non Empty Parameter Space for Indeterminacy) The two only possible regions for $\frac{\varphi_{1}}{\varphi_{2}}$, necessary and sufficient for having indeterminacy, are the following: i) $R_{D}^{0}<R_{14}^{0}<R_{15}^{0}<$ $R_{13}^{0}$ and ii) $R_{14}^{0}<R_{D}^{0}<R_{15}^{0}<R_{13}^{0}$.

Proof. $R_{D}^{0}<R_{13}^{0}$ stems directly from $1-\beta(1-\delta)<1$. Furthermore, $R_{14}^{0}$ is negative for all parameters' values and its denominator is always smaller than its numerator, as $2-\delta>1-\beta(1-\delta)$ (in absolute values). So it must be $R_{14}^{0}<R_{13}^{0}$. Lets now consider the following set of inequalities relative to the roots $R_{i}^{0}$.

Claim $4 R_{15}^{0}<R_{13}^{0}$.

\footnotetext{
${ }^{13}$ That are the intersections of the lines Numerator(13), Numerator(14), Numerator(15) and (common) Denumerator with the horizontal axis representing the values of $\frac{\varphi_{1}}{\varphi_{2}}$.
} 
Proof. Such inequality implies that $\frac{\delta s_{I}-\delta M \alpha(1+\eta)}{s_{I}(1-\beta(1-\delta))-\delta M \alpha(1+\eta)}>1$, and we first show that this can happen if and only if $s_{I}(1-\beta(1-\delta))<\delta M \alpha(1+\eta)$; in fact, if this is true, then it will be $\delta s_{I}<\delta M \alpha(1+\eta)$, as it is $1-\beta(1-\delta)>\delta$. When this happens, the numerator of $R_{15}^{0}$ is always negative and greater (in absolute value) than the denominator (which is also negative); then $\frac{\delta s_{I}-\delta M \alpha(1+\eta)}{s_{I}(1-\beta(1-\delta))-\delta M \alpha(1+\eta)}>1$. We show that the inequality $s_{I}(1-\beta(1-\delta))>\delta M \alpha(1+\eta)$ is incompatible with conditions (13)- (15), so that the opposite must hold. Suppose (by contradiction) that it is $s_{I}(1-\beta(1-\delta))>\delta M \alpha(1+\eta)$; then it would be either $\delta s_{I}>\delta M \alpha(1+\eta)$ or $\delta s_{I}<\delta M \alpha(1+\eta)$. In the first case the line Numerator(15) has a positive slope and a negative intersection $R_{15}$, which is greater than $R_{13}$; the situations is shown in figure 3.A). It is easy to check that for all values of $\frac{\varphi_{1}}{\varphi_{2}}$ inside the interval $\left(R_{D} ; R_{15}\right)$ indeterminacy never occurs, as $R_{D}$ is positive and $R_{15}$ is negative; but also for $\frac{\varphi_{1}}{\varphi_{2}}$ outside the interval indeterminacy never occurs, as condition (13) (for $\frac{\varphi_{1}}{\varphi_{2}}>R_{15}$ ) and condition (15) (for $\frac{\varphi_{1}}{\varphi_{2}}<R_{D}$ ) are not satisfied. Now consider the case in which $s_{I}(1-\beta(1-\delta))>\delta M \alpha(1+\eta)$ but $\delta s_{I}<\delta M \alpha(1+\eta)$; then Numerator(15) has still a positive slope but $R_{15}$ is positive. By shifting the line Numerator(15) in Figure 3.A), the same above argument applies and indeterminacy disappears. So, it must be $s_{I}(1-\beta(1-\delta))<\delta M \alpha(1+\eta)$, and then $R_{15}^{0}<R_{13}^{0}$.

Claim 5 We pass to show that $R_{D}^{0}<R_{15}^{0}$, i.e. that $\frac{\delta s_{I}-\delta M \alpha(1+\eta)}{s_{I}(1-\beta(1-\delta))-\delta M \alpha(1+\eta)}<\frac{1}{1-\beta(1-\delta)}$.

Proof. Note that when the term $\delta M \alpha(1+\eta)$ (which is always $\geqq 0$ ) is zero, the first fraction reduces to $\frac{1}{1-\beta(1-\delta)} \delta<\frac{1}{1-\beta(1-\delta)}$. We show that when the term $\delta M \alpha(1+\eta)$ increases, passing from zero to positive numbers, the fraction $\frac{\delta s_{I}-\delta M \alpha(1+\eta)}{s_{I}(1-\beta(1-\delta))-\delta M \alpha(1+\eta)}$ monotonically decreases, so that it must always be $R_{D}^{0}<R_{15}^{0}$. Consider $\delta M \alpha(1+\eta)$ as a function of $\eta$ : when $\eta$ is equal to ${ }^{14}$ -1 , the fraction collapses to $\frac{1}{1-\beta(1-\delta)} \delta$; when $\eta$ increases, the term $\delta M_{1} \alpha(1+\eta)$ monotonically increases. Now $\frac{d\left(-R_{15}\right)}{d \eta}=\frac{-\delta M \alpha}{s_{I}(1-\beta(1-\delta))-\delta M \alpha(1+\eta)}\left[1-\frac{\delta s_{I}-\delta M \alpha(1+\eta)}{s_{I}(1-\beta(1-\delta))-\delta M \alpha(1+\eta)}\right]$. We have seen before that $-\frac{\delta s_{I}-\delta M \alpha(1+\eta)}{s_{I}(1-\beta(1-\delta))-\delta M \alpha(1+\eta)}<-1$; but then it is $\frac{d\left(-R_{15}\right)}{d \eta}<0$ for all the parameters' values. Thus $R_{D}^{0}<R_{15}^{0}$.

Claim $6 R_{14}^{0}<R_{15}^{0}$.

Proof. We demonstrate this inequality by absurd. Assume that $R_{15}^{0}<R_{14}^{0}$; given the inequalities demonstrated above, two cases are possible: either $R_{14}^{0}<R_{D}^{0}$, or $R_{D}^{0}<R_{14}^{0}$; the first one is clearly impossible, as it would imply that $R_{15}^{0}<R_{14}^{0}<R_{D}^{0}$ and we have seen that it is $R_{D}^{0}<R_{15}^{0}$. Let's consider the second one: $R_{D}^{0}<R_{15}^{0}<R_{14}^{0}$; in this case the situation would be the one depicted in Figure 3.B) (recall that the slope of Numerator(14) is always positive). In the interval $\left(R_{14} ; R_{13}\right)$ indeterminacy is impossible, as Numerator $(15)<0$ and Denominator $>0$; this is also true in the interval $\left(R_{D} ; R_{14}\right)$, as Numerator $(14)<0$, Denominator $>0$, and in the regions outside the two intervals. Then the only ordering compatible with indeterminacy is $R_{14}^{0}<R_{15}^{0}$.

Claim 7 The last step is to show that both $R_{14}^{0}<R_{D}^{0}$ and $R_{D}^{0}<R_{14}^{0}$ are possible and compatible with an interval for indeterminacy (although for our parametrization the case is $R_{D}^{0}<R_{14}^{0}$ ).

Proof. The order between $R_{14}^{0}$ and $R_{D}^{0}$ does not affect the existence of a non empty parameter space for indeterminacy of equilibrium, as Figure 3.C) illustrates. The interval $\left(\max \left\{R_{D}^{0}, R_{14}^{0}\right\} ; R_{15}\right)$ is

\footnotetext{
${ }^{14}$ Obviously $\eta<0$ is not an interesting case in our model (it could be interpreted as a negative externality at system level), but for the sake of the argument it can be accepted just to see what is the effect on the fraction of the term $\delta \alpha(1+\eta) M$ when the latter is arbitrary small.
} 
a viable region for indeterminacy, as for all the $\frac{\varphi_{1}}{\varphi_{2}}$ in this region, the three conditions (13)-(15) are satisfied.

Summing up, we have demonstrated that: $R_{D}^{0}<R_{13}^{0}, R_{14}^{0}<R_{13}^{0}, R_{15}^{0}<R_{13}^{0}, R_{D}^{0}<R_{15}^{0}, \quad R_{14}^{0}<$ $R_{15}^{0}, R_{14}^{0}<R_{D}^{0}$ or $R_{D}^{0}<R_{14}^{0}$. By merging all these inequalities together, the orderings compatible with an indeterminacy region turn out to be $R_{D}^{0}<R_{14}^{0}<R_{15}^{0}<R_{13}^{0}$ and/or $R_{14}^{0}<R_{D}^{0}<R_{15}^{0}<R_{13}^{0}$.

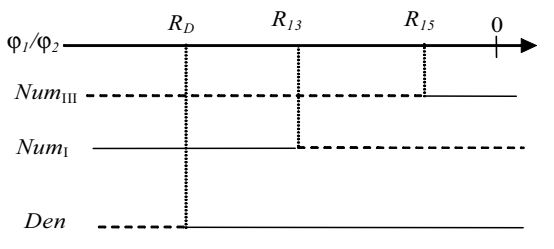

A)

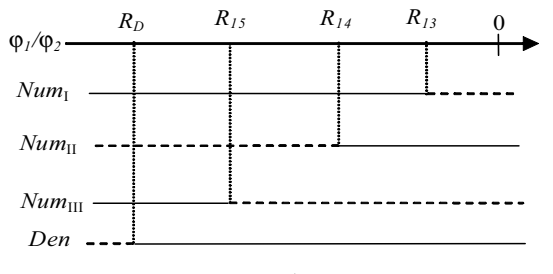

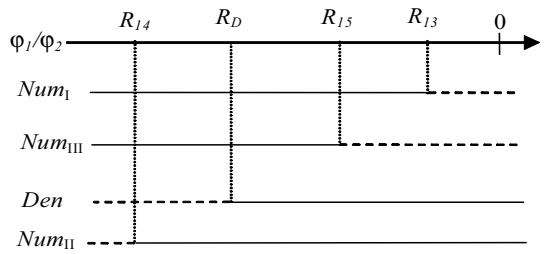

C)

Figure 3: Auxiliary intervals: dotted lines represent negative values of the corresponding function of $\frac{\varphi_{1}}{\varphi_{2}}$, (i.e. the three numerators of Conditions (2) - (4) and the common denumerator) while solid lines represent positive values.

So we have two possible orderings defining the indeterminacy region; one is given by the interval $\left(R_{14}^{0} ; R_{15}^{0}\right)$, or

$$
-\underline{\mathcal{R}}<\frac{\varphi_{1}}{\varphi_{2}}<-\overline{\mathcal{R}}
$$

where:

$$
\begin{aligned}
& \underline{\mathcal{R}}=\frac{\delta\left(M-s_{I}\right)[1-\beta(1-\delta)](1-\alpha(1+\eta))+2\left[\delta \alpha(1+\eta) M+s_{I}(2-\delta)\right]}{\delta\left(M-s_{I}\right)[1-\beta(1-\delta)](1-\alpha(1+\eta))+2\left[\delta \alpha(1+\eta) M+s_{I}(1-\beta(1-\delta))\right]} \\
& \overline{\mathcal{R}}=\frac{\delta s_{I}-\delta M \alpha(1+\eta)}{s_{I}(1-\beta(1-\delta))-\delta M \alpha(1+\eta)}
\end{aligned}
$$

The other one is given by $\left(R_{D}^{0}, R_{15}^{0}\right)$, or:

$$
-\frac{1}{1-\beta(1-\delta)}<\frac{\varphi_{1}}{\varphi_{2}}<-\overline{\mathcal{R}}
$$

ordering (17) is clearly more useful for finding an economic interpretation of the conditions for indeterminacy. Basically, both the previous equations tell us that in order to have indeterminacy, the ratio $\frac{\varphi_{1}}{\varphi_{2}}$ must be negative, greater (in modulus) than one and finally included between two specific values. We can use the "easier" interval (17) without great loss of generality. From the above theorem, we can see that the necessary condition for indeterminacy ${ }^{15}$ is given by:

$$
s_{I}(1-\beta(1-\delta))<\delta M \alpha(1+\eta)
$$

which, after some algebraic manipulation, turns out to be condition NC. As for condition GC, note that ratio $\frac{\varphi_{1}}{\varphi_{2}}$ is given by $\frac{\varphi_{1}}{\varphi_{2}}=\frac{(1+\zeta) \rho+(1+\eta)(1-\alpha-\rho)}{1-(1+\zeta) \rho-(1+\eta)(1-\alpha-\rho)}<-1\left(=R_{13}^{0}\right) .{ }^{16}$ Putting toghether (16) and (17) and explicitating with respect to $\varphi_{1}$, condition $\mathbf{G C}$ in the main text can be obtained.

\footnotetext{
${ }^{15}$ Related to the absolute value of the determinant of matrix $F$, i.e. to the product of the two eigenvalues.

${ }^{16}$ Note also that the term $\varphi_{1}$ is equal to sum of the cross elasticities of the two labor demands: $\varepsilon_{\widehat{K}}^{\widehat{L}_{M}^{D}}$ and $\varepsilon_{\widehat{K}}^{\widehat{L}_{U}^{D}}$, as shown in the main text
} 